\title{
Relativistic particle acceleration in an electron-positron plasma with a relativistic electron beam
}

\author{
J. Zhao and J. I. Sakai \\ Department of Electronics and Information, Toyama University, Toyama, 930 Japan \\ K.-l. Nishikawa \\ Department of Physics and Astronomy, The University of lowa, lowa City, lowa 52242 \\ T. Neubert \\ Space Physics Research Laboratory, The University of Michigan, Ann Arbor, Michigan 48109
}

(Received 15 July 1994; accepted 6 September 1994)

\begin{abstract}
Results from three-dimensional electromagnetic particle simulations of an electron-positron plasma with a relativistic electron beam $(\gamma=2)$ are presented. As part of the initial conditions, a poloidal magnetic field is specified, consistent with the current carried by the beam electrons. The beam undergoes pinching oscillations due to the pressure imbalance. A transverse two-stream instability is excited with large helical perturbations. In the process, background electrons and positrons are heated and accelerated up to relativistic energy levels. Only background electrons are accelerated farther along the $z$ direction due the synergetic effects by both the damped transverse mode and the accompanying electrostatic waves caused by the breakdown of the helical perturbations. (C) 1994 American Institute of Physics.
\end{abstract}

\section{INTRODUCTION}

It is known that magnetized relativistic electron-positron plasmas exist in the region of the pulsar magnetosphere and in active galactic nuclei (AGN). The problem with strong electromagnetic waves in electron-positron plasmas is of prime importance in understanding the emission processes in pulsars, and has been the subject of several investigations. ${ }^{1-5}$ Such electromagnetic waves are capable of driving plasma particles to relativistic energies. This energetic particle distribution may account for the periodic radiation observed in association with pulsars. ${ }^{6-8}$ It has been shown in many cases that when the field amplitudes are large, the waves suffer modulational or filamentational instabilities, ${ }^{9,10}$ and may become broken into highly localized pulses or filaments, which could be associated with observed pulsar radiation. However, all these studies are restricted to the theoretical analysis of wave propagation and linear dispersion relations. Since nonlinear effects in the magnetized relativistic plasma may play an important role in wave generation, interpretations considering only linear effects are not sufficient. Thus, we chose to do computer simulations to investigate some processes of an electron-positron plasma. In the study of a nonrelativistic electron beam in an electron-positron plasma, Zhao et al. ${ }^{11}$ has shown that bunching of the beam due to the beam instability is directly involved in the generation of Alfvén waves. Alfvén waves propagate along the beam as damped solitons accelerating the background particles.

Recently, relativistic electron-heam instabilities have been studied for several applications. The "electron-hose" instability was proved to be excited by a relativistic electron beam in an unmagnetized plasma. ${ }^{12}$ Intensive studies on the two-stream instability have been done, in connection with its application to a high-power relativistic klystron amplifier (RKA). ${ }^{13-16}$

In this paper, we confine ourselves to carry out numcrical simulations on a relativistic electron beam in an electron-positron plasma by a three-dimensional electromagnetic particle code. ${ }^{17-20}$ The simulation results show that a transverse two-stream instability ${ }^{21}$ (e.g., the "electronhose" instability) takes place during the beam is undergoing pinching oscillations. The particles are heated and accelerated to relativistic energies associated with the growth of the transverse two-stream instability, and only the background electrons are further accelerated by both the damped transverse mode and electrostatic waves produced by the breakdown of the helical perturbations.

\section{SIMULATION RESULTS}

\section{A. System model and parameters}

The code is a three-dimensional (3-D), fully electromagnetic, and relativistic particle code. The domain is a mesh of size $85 \Delta \times 85 \Delta \times 160 \Delta$, where $\Delta(=1)$ is the grid size. Periodic boundary conditions are used for particles and fields in the $z$ direction, while radiating boundary conditions ${ }^{22}$ are used in the $x$ and $y$ directions. Particles that hit these boundaries are arrested there and then reflected with a thermal velocity randomly with some portion (50\%). There are 1000000 electron-positron pairs filling the entire domain uniformly and that keeps the domain charge neutral.

An electron beam in the $z$ direction is specified initially in a column with radius $r_{\mathrm{eb}}=4.47 \Delta$ at the center of the $x y$ plane $\left(x_{c n}=43, y_{c n}=43\right)$. Within the column, half the electrons are beam electrons, while the other half are background electrons. The total number of beam electrons is 10000 . The temperature of the beam electrons is much colder than the temperature of the background electrons $\left(T_{\mathrm{eb}}=0.09 T_{e}\right)$. Initially, only the beam electrons drift with $v_{d}=0.865 c$ ( $c$ is the light velocity), corresponding to a Lorentz factor $\gamma=\left[1-\left(v_{d} / c\right)^{2}\right]^{-1 / 2}=2$. In addition to a homogeneous background magnetic field $B_{z 0}$, a poloidal magnetic field $B_{\theta}$, consistent with the current density $J_{z}$ carried by the beam electrons, is included initially ${ }^{20}$ with $B_{\theta} / B_{z}=0.48$. Other pa- 

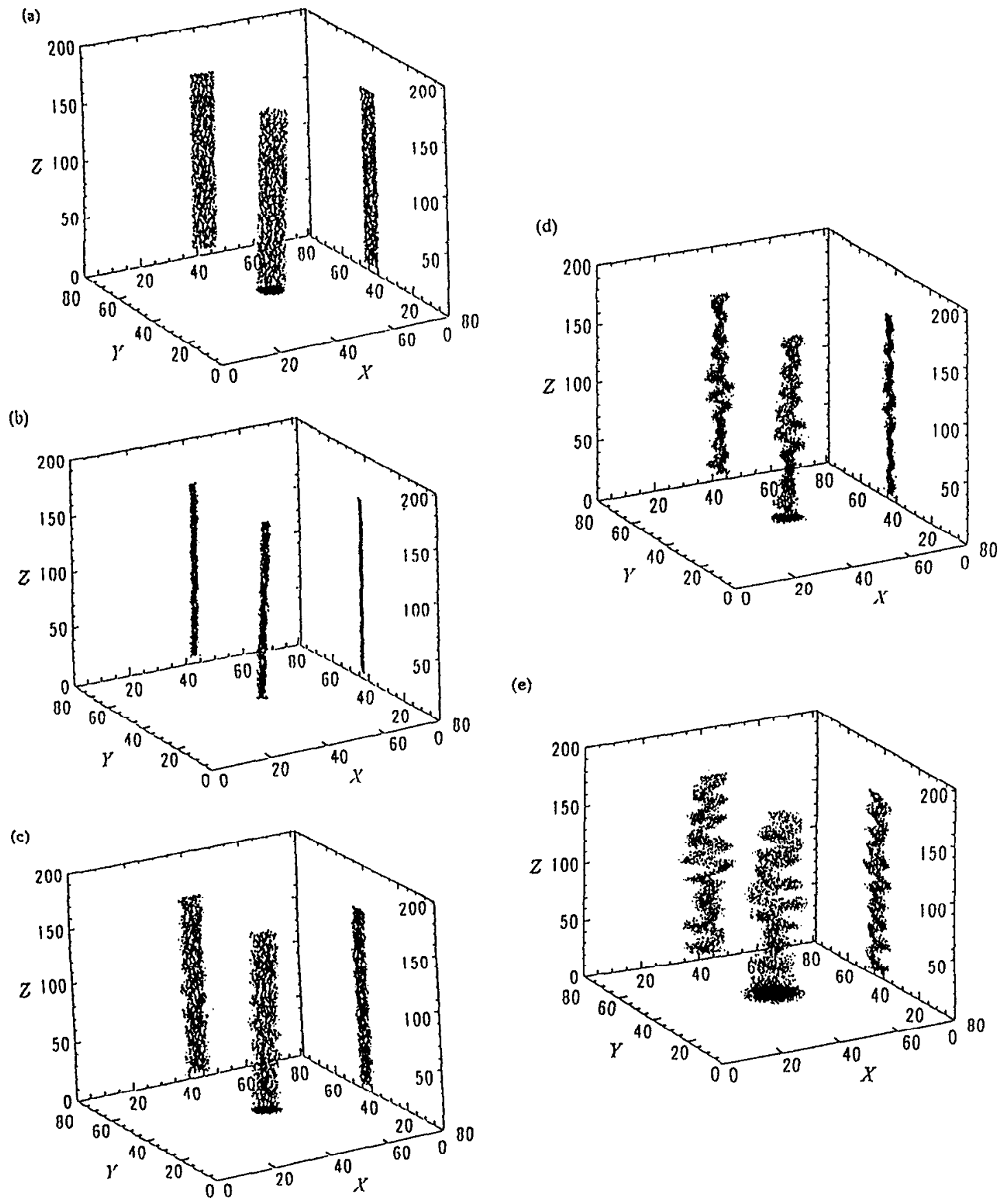

(e)



FIG. 1. The beam electrons shown in a three-dimensional space with the projections onto the $x-y, x-z$, and $y-z$ planes at $\omega_{p c} t=0$ (a), 5 (b), 10 (c), 15 (d), and $20(\mathrm{e})$.

rameters are as follows: $m_{p} / m_{e}=1, T_{e} / T_{p}=1\left(T_{p}\right.$ is the positron temperature), $\Omega_{e} / \omega_{p e}=0.4, c / v_{t g}=10.67\left(v_{t g}\right.$ is the thermal velocity of the background electrons), $\omega_{p e} \Delta t=0.1$, $\beta=0.111, \lambda_{D e}=0.469 \Delta, c / \omega_{p e}=5.0 \Delta$, and the gyroradii of electrons and positrons are $\rho_{e}=\rho_{p}=1.17 \Delta$.

\section{B. Excitation of a transverse two-stream instability with helical perturbations}

Figure 1 plots time evolution of the beam electrons in a three-dimensional space, clearly showing that the beam be- gins to undergo pinching oscillations first. After that, it is disturbed with helical perturbations. Because the initial drift velocity is very large, the current density $J_{z}$ carried by the beam electrons becomes large. The poloidal magnetic field associated with the beam current causes a pressure imbalance between the inside and outside of the beam, which, in turn, drives periodical pinching of the whole beam. This pinching effect plays an important role in increasing the betatron oscillations. The beam electrons do large-amplitude betatron orbits that span the pinched electron beam. Therefore, the 


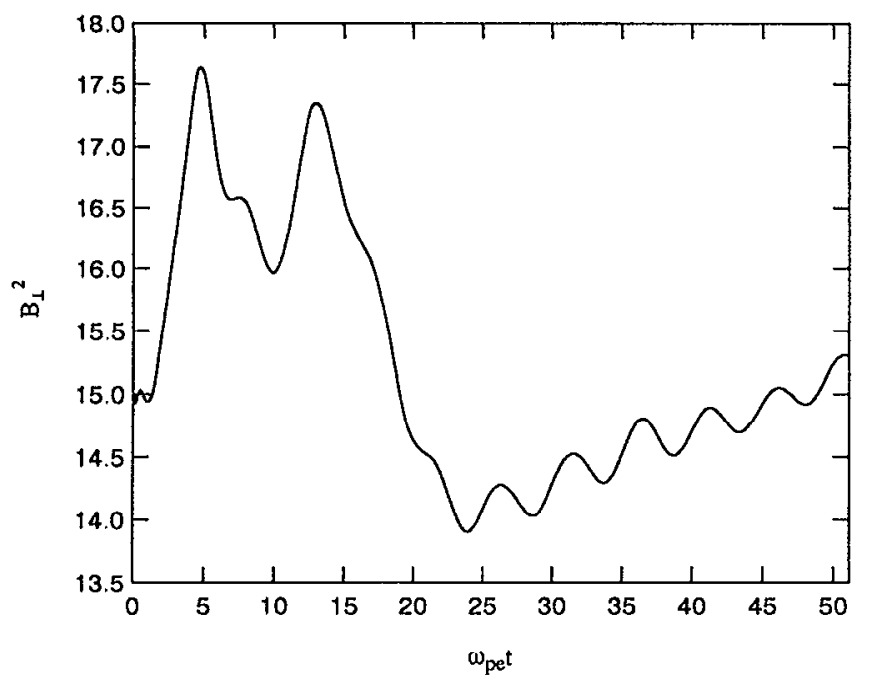

FIG. 2. Time evolution of the perpendicular magnetic field energy.

beam electrons are easily transversely displaced, due to the sheared helical total magnetic energy, which is an energy source of a transverse two-stream instability (TTSI). This instability results in helical perturbations of the beam with a release of the perpendicular magnetic energy rapidly, as seen in Fig. 2, showing that $B_{\perp}^{2}$ decreases after $\omega_{p e} t=12$. The time evolution of the magnetic field component $B_{x}$ along the beam axis is illustrated in Fig. 3. It is clearly seen that waves are excited after the occurrence of the instability, propagating along the beam direction. The waves own a right-hand polarization, as shown in Fig. 4.

\section{Particle heating and acceleration}

Figures 5 and 6 display velocity distributions of the beam electrons, background electrons, and positrons at $\omega_{p e} t=0$ (dashed line) and $\omega_{p e} t=20$ (solid line) in the $x$ and $z$ directions, respectively. It is clearly seen that the back-

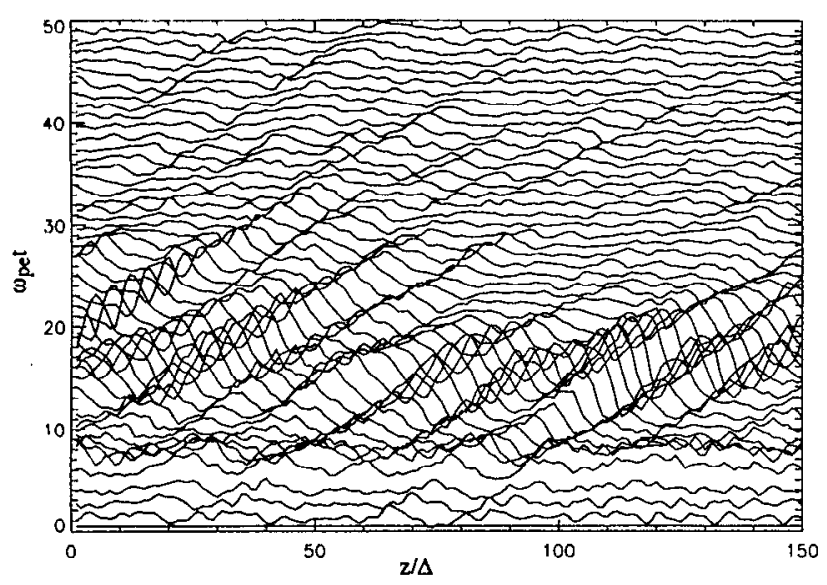

FIG. 3. Time history of $B_{x}$ along the $z$ direction in the center of the beam $(x=43, y=43)$. (a)

|ง

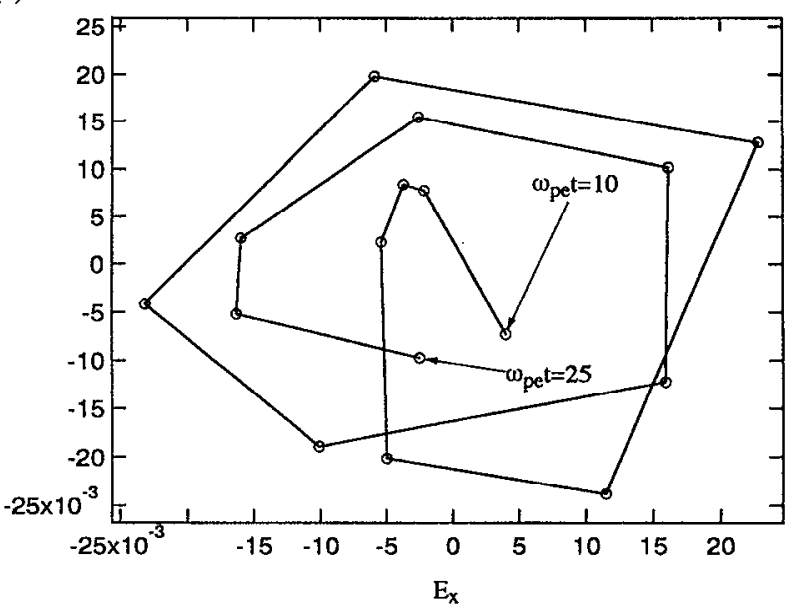

(b)

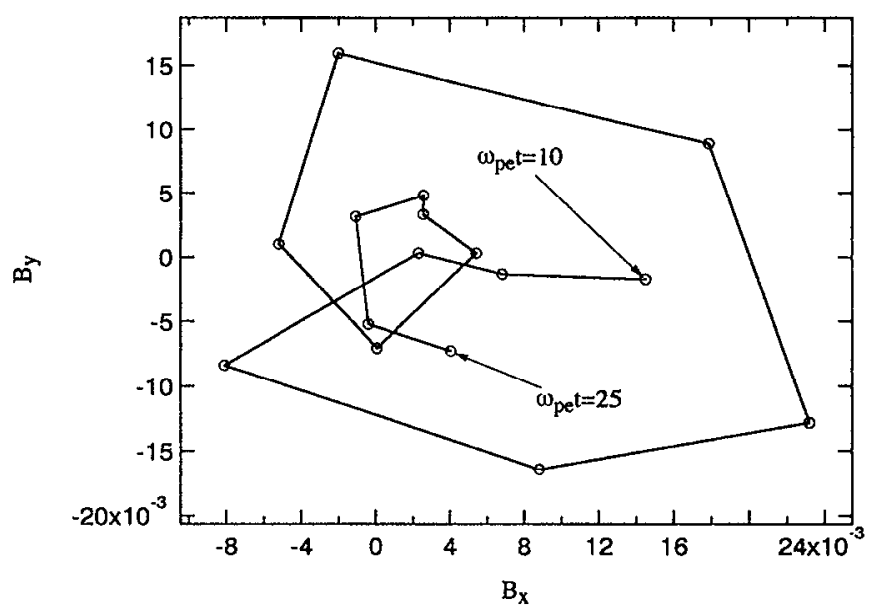

FIG. 4. Time history of $E_{x}$ vs $E_{y}$ (a) and $B_{x}$ vs $B_{y}$ (b) taken at the point $(x=43, y=43$, and $z=85)$.

ground electrons and positrons are not only heated up to relativistic energies in the transverse directions with a maximal $\gamma$ about 1.1, but also accelerated in the $z$ direction with a maximal $\gamma$ about 1.2. At the end of the simulation $\left(\omega_{p e} t=50\right)$, the distribution of $v_{x}$ shows no further modification, indicating that particle heating in the transverse direction mainly retains to the phase of excitation of the TTSI.

Figure 7 shows snapshots of the phase space distribution of the beam electrons at $\omega_{p e} t=25$. One can see that velocities of the beam electrons in the $z$ direction are characterized by the vortex turbulence, and that the beam electrons are helically bunched in the $x-z$ space. These bunches create the electric field in the $z$ direction, which increases rapidly and becomes the dominant component of the electric field. Later, in the final stage, it damps out. Figure 8 shows the intensity of the charge density fluctuations for the last 256 time steps of the simulation, corresponding to the time range of $25 \omega_{p e}^{-1}$ to $50 \omega_{p e}^{-1}$ by performing a two-dimensional Fourier transformation (one space and one time). The gray scale is logarithmic in the wave intensity, and is arbitrary. As seen in Fig. 8, electrostatic waves are excited with a dominant frequency near the electron plasma frequency, and the primary wave- 
(a)

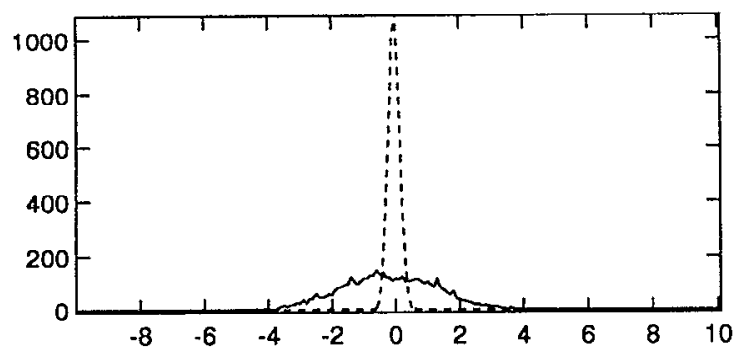

(b)

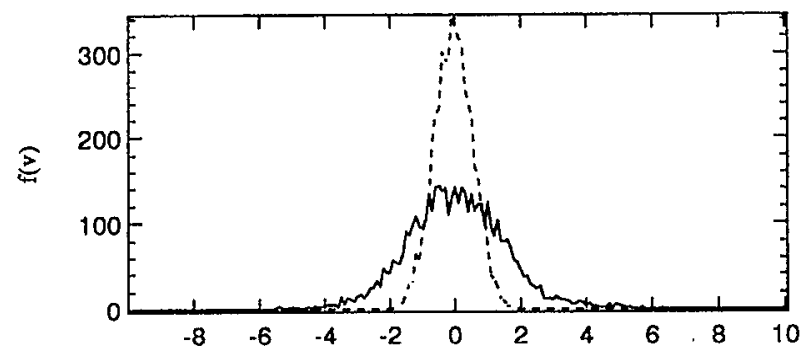

(c)

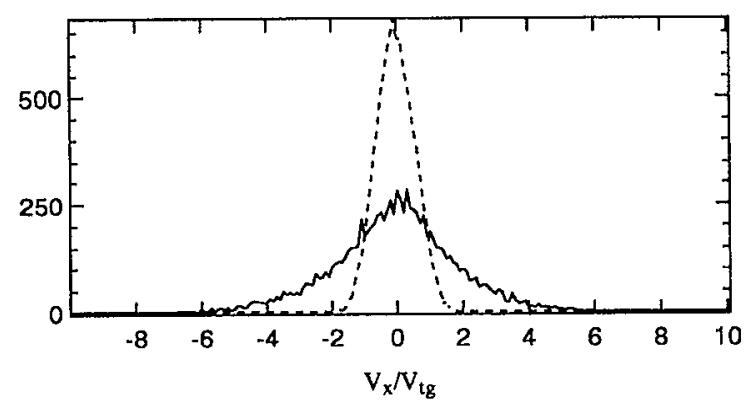

FIG. 5. Velocity distributions of the particles initially inside the column in the $x$ direction at $\omega_{p e} t=0$ (dashed line) and $\omega_{p e} t=20$ (solid line). (a) The beam electrons; (b) the background electrons; and (c) the positrons.

length is approximately estimated at $18 \Delta$, which is in accord with the pitch of the helical perturbations. We interpret the excitation of bunching as that the helical perturbations break down, resulting in the beam electrons to be bunched along the $z$ direction. Therefore, the excited electrostatic wave number is seemed to be set up by the TTSI.

Velocity distributions of the particles inside the column in the $z$ direction at $\omega_{p e} t=50$ are shown in Fig. 9. The background electrons have been further accelerated to higherenergy levels associated with a maximal $\gamma$ of the order of 1.86. However, the positrons are almost unaffected. The fact that the excited waves are right-hand polarized means that they are electron dominated, therefore the electrons are preferably accelerated. From Fig. 3 , it is seen that the excited transverse mode begins to damp after $\omega_{p e} t=20$, while at the same time, strong electrostatic waves are excited by the bunched beam electrons. The background electrons are probably accelerated along the $z$ direction by both the damped (a)

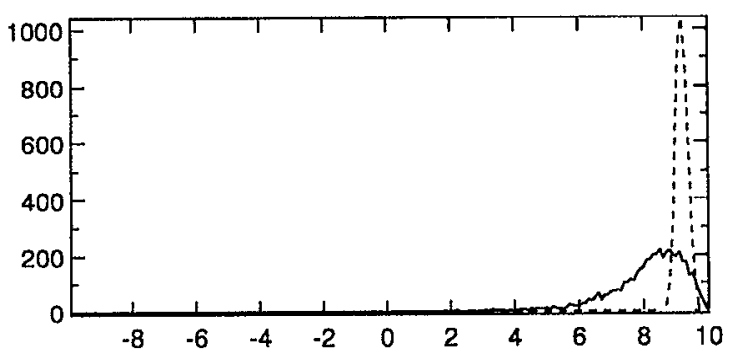

(b)

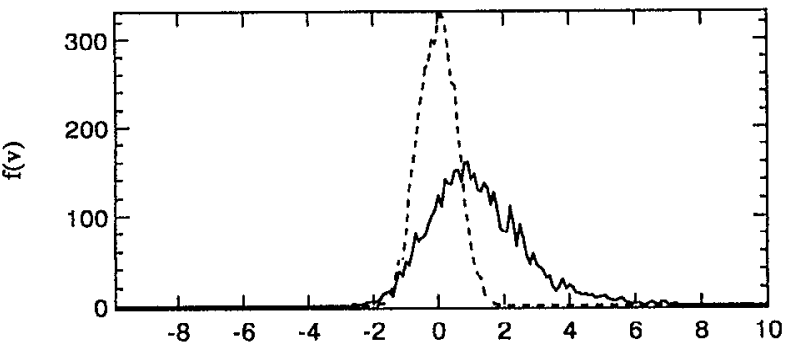

(c)

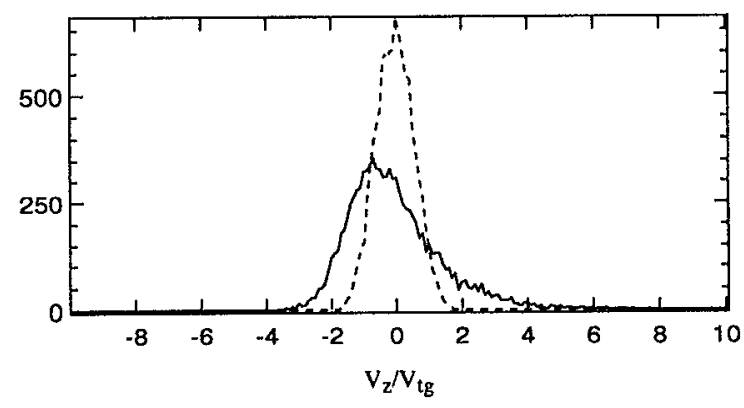

FIG. 6. Velocity distributions of the particles initially inside the column in the $z$ direction at $\omega_{p e} t=0$ (dashed line) and $\omega_{p e} t=20$ (solid line). (a) The beam electrons; (b) the background electrons; and (c) the positrons.

transverse mode and the electrostatic waves, which is the same mechanism elucidated in Ref. 11.

\section{SUMMARY}

Wc have presented simulation results of a relativistic electron beam in an electron-positron plasma using a 3-D electromagnetic particle. The beam undergoes pinching oscillations due to a pressure imbalance caused by the strong poloidal magnetic field generated initially by the electron beam. A transverse two-stream instability is excited with the helical perturbations because the pinching makes the beam electrons do large-amplitude betatron orbit, which spans the beam radius. After breakdown of the helical perturbations, the bcam electrons are bunched in the $z$ direction, accompanying excitation of the electrostatic waves. The background electrons and positrons are heated and accelerated up to relativistic energies associated with the excited transverse mode. Only the background electrons are further accelerated along the $z$ direction by the damped transverse mode and the electrostatic waves produced by the bunched beam electrons. 
(a)

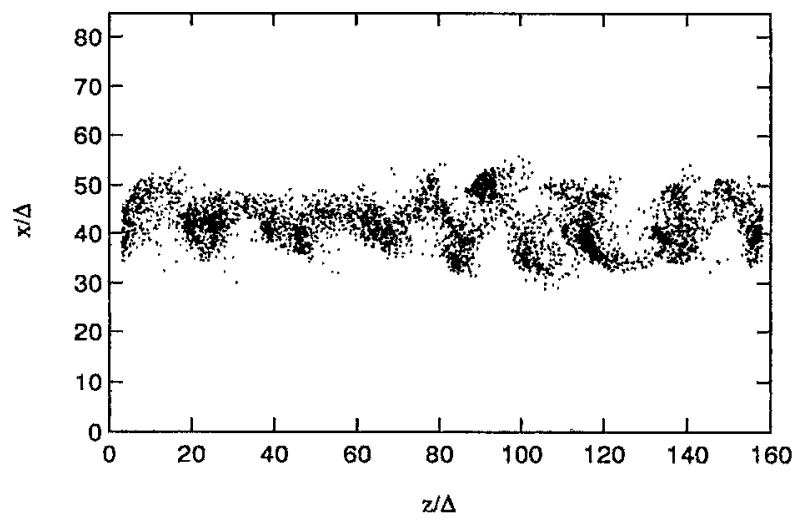

(b)



FIG. 7. Snapshots of the phase space distributions of the beam electrons at $\omega_{p e} t=25$. (a) The phase space in $z-x$; and (b) the phase space in $z-v_{z}$.

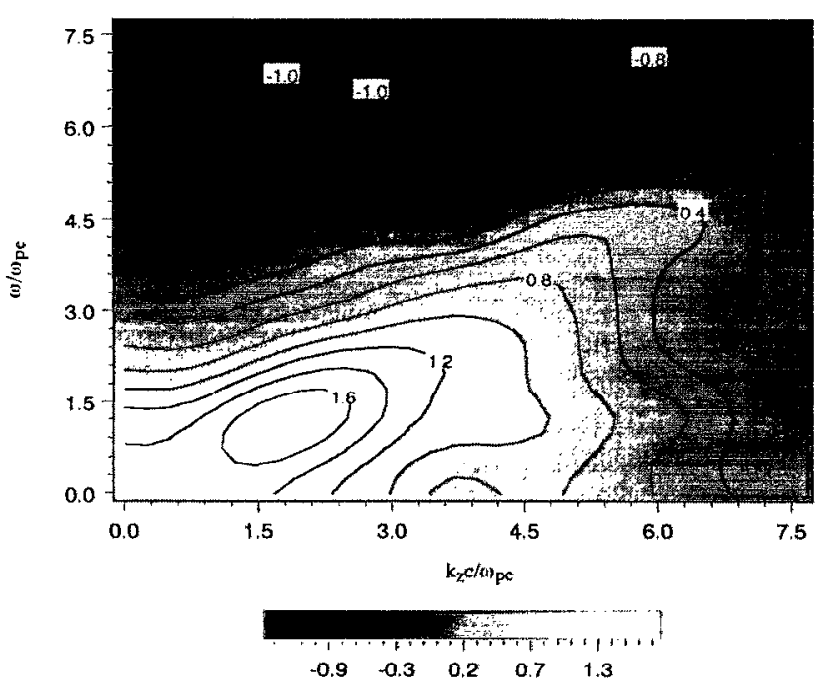

FIG. 8. Intensity of the charge density oscillation along the $z$ direction in the center of the beam $(x=43, y=43)$. (a)



(b)

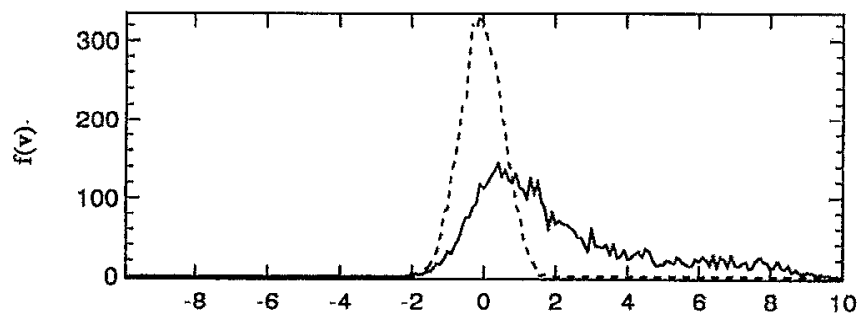

(c)

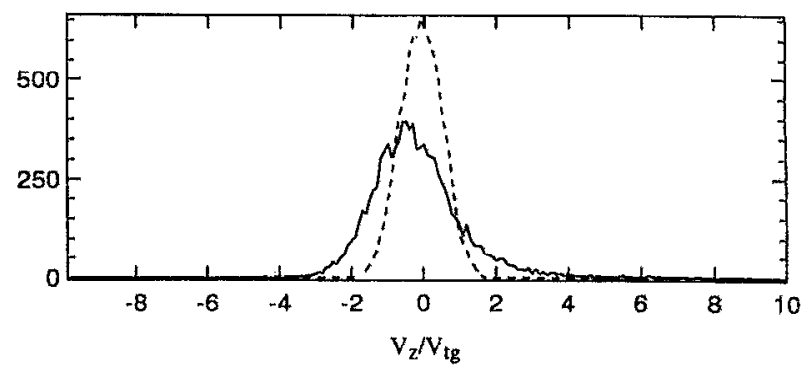

FIG. 9. Velocity distributions of the particles initially inside the column in the $z$ direction at $\omega_{p e} t=0$ (dashed line) and $\omega_{p e} t=50$ (solid line). (a) The beam electrons; (b) the background electrons; and (c) the positrons.

\section{ACKNOWLEDGMENTS}

We appreciate the constructive comments and suggestions of the reviewers on the previous version of this paper. The simulations were performed at the National Center for Supercomputing Applications, University of Illinois at Urbana-Champaign supported by the NSF foundation and on the mini-super computer "ALLIANT" located at Toyama University.

This work was carried out under the auspices of the U. S.-Japan cooperative science program of Japan Society for the Promotion of Science and the National Science Foundation (NSF) (INT-9217650). J. Zhao is grateful to the Japanese Education Ministry for a scholarship. K.-I. Nishikawa is supported by the NSF under Grants No. ATM-9119814 and No. ATM-912116. T. Neubert is supported by the National Aeronautics and Space Administration under Contract No. NAS-38772.

${ }^{1}$ J.-I. Sakai and T. Kawata, J. Phys. Soc. Jpn. 49, 753 (1980).

${ }^{2}$ A. B. Mikhailoskii, O. G. Onishchenko, and A. I. Smolyakov, Sov. I. Plasma Phys. 11, 215 (1985).

${ }^{3}$ L. Stenflo, P. K. Shukla, and M. Y. Yu, Astron. Space Sci. 117, 303 (1985). 
${ }^{4}$ M. Y. Yu, K. Shukla, and L. Stenflo, Astrophys. J. 309, 63 (1986).

${ }^{5}$ R. Bhruthram, P. K. Shukla, and M. Y. Yu, Astron. Space Sci. 135, 211 (1987).

${ }^{6}$ C. E. Max, Phys. Fluids 16, 1277 (1973).

${ }^{7}$ C. F. Kemel, F. S. Fujimura, and R. Pellat, Space Sci. Rev. 24, 407 (1979).

${ }^{8}$ A. C.-L. Chian, Astron. Astrophys. 112, 391 (1982).

${ }^{9}$ A. C.-L. Chian and C. F. Kennel, Astron. Space Sci. 97, 9 (1983).

${ }^{10}$ P. K. Shukla, Astron. Space Sci. 114, 381 (1985).

"J. Zhao, K. I. Nishikawa, J. I. Sakai, and T. Neubert, Phys. Plasmas 1, 103 (1994).

${ }^{12}$ D. H. Whittum, W. M. Sharp, S. S. Yu, M. Lampe, and G. Joyce, Phys. Rev. Lett. 67, 991 (1991).

${ }^{13}$ H. S. Uhm, G. S. Park, and C. M. Armstrong. Phys. Fluids B 5, 1349 (1993).

${ }^{14}$ C. Chen, P. Catravas, and G. Bekefi, Appl. Phys. Lett. 62, 1579 (1993).
${ }^{15}$ H. S. Uhm and C. Chen, Phys. Fluid B 5, 4180 (1993).

${ }^{16}$ H. S. Uhm, Phys. Fluids B 5, 3388 (1993).

${ }^{17}$ O. Buneman, in Computer Space Plasma Physics, Simulation Techniques and Softwares, edited by H. Matsumoto and Y. Omura (Terra Scientific, Tokyo, 1993), p. 67.

${ }^{18}$ O. Buneman, T. Neubert, and K.-I. Nishikawa, IEEE Trans. Plasma Sci. PS-20, 810 (1992).

${ }^{19}$ T. Neubert, R. H. Miller, O. Buneman, and K.-I. Nishikawa, J. Geophys. Res. 97, 12057 (1992).

${ }^{20}$ K. I. Nishikawa, O. Bunernan, and T. Neubert, Geophys. Res. Lett. 21, 1019 (1994).

${ }^{21} \mathrm{~J}$. D. Lawson, The Physics of Charged-Particle Beams (Oxford University Press, London, 1977), pp. 384-387.

${ }^{22}$ E. L. Lindman, J. Comput. Phys. 18, 66 (1975). 